\title{
Analysis of the Bacterial Diversity Associated with the Roots of Maize (Zea mays L.) through Culture-Dependent and Culture-Independent Methods
}

\author{
Paola Pereira, ${ }^{1}$ Fernando Ibáñez, ${ }^{2}$ Mónica Rosenblueth, ${ }^{3}$ \\ Miriam Etcheverry, ${ }^{1}$ and Esperanza Martínez-Romero ${ }^{3}$ \\ ${ }^{1}$ Departamento de Microbiología e Inmunología, Facultad de Ciencias Exactas, Físico-Químicas y Naturales, \\ Universidad Nacional de Río Cuarto, Consejo Nacional de Investigaciones Científicas y Técnicas (CONICET), \\ Ruta Nac. 36 Km 601, Río Cuarto, 5800 Córdoba, Argentina \\ ${ }^{2}$ Departamento de Ciencias Naturales, Facultad de Ciencias Exactas, Físico-Químicas y Naturales, \\ Universidad Nacional de Río Cuarto, Consejo Nacional de Investigaciones Científicas y Técnicas (CONICET), \\ Ruta Nac. 36 Km 601, Río Cuarto, 5800 Córdoba, Argentina \\ ${ }^{3}$ Centro de Ciencias Genómicas, Programa de Ecología Genómica, Universidad Nacional Autónoma de México, \\ Apartado Postal 565-A, Av. Universidad s/n., Col. Chamilpa, C.P. 62210, Cuernavaca, Morelos, Mexico
}

Correspondence should be addressed to Esperanza Martínez-Romero, emartine@ccg.unam.mx

Received 19 January 2011; Accepted 24 February 2011

Academic Editor: M. A. Molina-Montenegro

Copyright () 2011 Paola Pereira et al. This is an open access article distributed under the Creative Commons Attribution License, which permits unrestricted use, distribution, and reproduction in any medium, provided the original work is properly cited.

The present study investigated bacterial diversity associated with the roots of maize through the use of culture-dependent and culture-independent methods. Bacterial 16S-23S rDNA internal transcribed spacer sequences (ITS) primers were used to amplify sequences obtained directly from the root matrix by Percoll gradient separation. This assay showed that $\gamma$-Proteobacteria within Enterobacter, Erwinia, Klebsiella, Pseudomonas, and Stenotrophomonas genera were predominant groups. The culturable component of the bacterial community was also assessed, revealing that the predominant group was Firmicutes, mainly of Bacillus genus, while Achromobacter, Lysinibacillus, and Paenibacillus genera were rarely found in association with the roots. Only two genera within $\gamma$-Proteobacteria, Enterobacter and Pseudomonas, were found in the culture collection. Differences in richness and diversity between the rhizospheric and endophytic bacterial communities were also evidenced. The spectrum of bacteria naturally associated with maize roots is wide and the magnitude of such diversity will depend on the methods chosen for analysis. The knowledge of this spectrum will facilitate the search of microorganisms capable of exerting antagonism to diverse pathogens or detecting plant growth enhancers.

\section{Introduction}

Maize (Zea mays L.) is one of the three most important agronomic crops in terms of world production, together with rice and wheat. As world cereal consumption tends to increase due to a constantly growing population, productivity should be significantly improved through different strategies that allow an optimization of yields without implicating an increased sown area [1]. Despite the partial success of synthetic chemical pesticides and fertilizers in achieving this goal, a change to environmentally friendly and conservative alternatives is required to protect biodiversity and sustainability of agroecosystems and natural systems all over the world.

Soil microbial communities play an integral and often unique role in ecosystem functions and are among the most complex, diverse, and important assemblages in the biosphere [2]. The study of plant-associated microorganisms is of great importance for biotechnological applications, for example, biological control of plant pathogens, plant growth promotion, or isolation of active compounds $[3,4]$.

Most studies on rhizospheric and endophytic bacteria and their community structure have been performed by 
using culture-dependent approaches. Isolation of culturable bacteria is appropriate for functional analysis; however, as a high percentage of naturally occurring bacteria remains in a nonculturable state $[5,6]$ the use of culture-independent methods provides additional information on the diversity of bacterial communities [7]. Previous studies have analyzed bacterial taxa associated with maize. Some of these studies have been focused on the culturable fraction [8-10] while others assessed bacterial diversity independently of culture [11-14]. However, none of these studies provided an analysis of rhizospheric and endophytic bacterial diversity assessed by both culture-dependent and culture-independent methods. The present work was carried out to gain insight into bacterial diversity associated with the roots of maize seedlings by culture-dependent and culture-independent approaches.

\section{Materials and Methods}

2.1. Plant Material and Sampling. Grains of maize cv. Monsanto DK684RR2 were sown in plastic pots, $10 \mathrm{~cm}$ in diameter $\times 15 \mathrm{~cm}$ high, filled with sandy loam soil obtained from an Argentinean maize field $(\mathrm{pH} 6.1$ in water $1: 1 \mathrm{w} / \mathrm{v}$, $1.4 \%$ organic matter, $86 \mathrm{ppm}$ of nitrates).

All pots were irrigated with sterile distilled water once immediately after sowing to half full soil water holding capacity. Pots with seeds were then incubated with $12 \mathrm{~h}$ photoperiod and at $28 \pm 2^{\circ} \mathrm{C}$ until sampling.

Twenty days after sowing, ten seedlings were harvested and their root systems were aseptically separated from the shoots for further analysis. Roots were processed immediately after sampling.

\subsection{Analysis of Bacterial Diversity by Culture-Dependent Methods}

2.2.1. Isolation and Number Estimation of Bacteria from Maize Roots. Roots of harvested plants (average weight of $3 \mathrm{~g}$ ) were individually submerged in a volume of sterile phosphatebuffered saline (PBS: $\mathrm{NaCl} 8 \mathrm{~g}, \mathrm{KCl} 0.2 \mathrm{~g}, \mathrm{Na}_{2} \mathrm{HPO}_{4} 1.15 \mathrm{~g}$, $\mathrm{KH}_{2} \mathrm{PO}_{4} 0.2 \mathrm{~g}$, deionized water $1000 \mathrm{ml}, \mathrm{pH}$ 7.3) obtaining a $1 / 10$ dilution of the sample. Roots were vigorously vortexed for $5 \mathrm{~min}$ to separate adherent soil and serial 10-fold dilutions were prepared in sterile PBS up to $10^{-8}$. Aliquots of $0.1 \mathrm{ml}$ were taken from the different dilutions and plated in duplicate on nutrient agar (NA: meat extract $3 \mathrm{~g}$, soy peptone $5 \mathrm{~g}, \mathrm{NaCl} 8 \mathrm{~g}$, agar-agar $15 \mathrm{~g}$ ) to count colony forming units (CFUs) of the rhizoplane. Afterwards, roots were washed with sterile distilled water and surface-sterilized by immersion in $1.3 \%$ sodium hypochlorite $(15 \mathrm{~min})$ followed by four rinses with sterile distilled water, an immersion in $2 \%$ sodium thiosulfate to remove the residual sodium hypochlorite [15] and, a final rinse with sterile distilled water. Surface sterilization was considered to be achieved by the absence of CFUs in NA plates. Roots were then placed into a chilled sterile mortar under sterile conditions and macerated with a saline buffer $\left(50 \mathrm{mM} \mathrm{KH} \mathrm{PO}_{4}, 150 \mathrm{mM}\right.$ $\mathrm{NaCl}, \mathrm{pH}: 7.6)$ cooled at $4^{\circ} \mathrm{C}$ to isolate bacteria from the root
TAble 1: Primer pairs used for the amplification of $16 \mathrm{~S}$ rRNA sequences (a) and 16S-23S rRNA spacer sequences (b).

\begin{tabular}{|c|c|c|c|}
\hline & Primer & Sequence $\left(5^{\prime}\right.$ to $\left.3^{\prime}\right)$ & Reference \\
\hline \multirow{2}{*}{ (a) } & $\mathrm{fD} 1$ & AGAGTTTGATCCTGGCTCAG & \multirow{2}{*}{ Weisburg et al. [16] } \\
\hline & rD1 & AAGGAGGTGATCCAGCC & \\
\hline \multirow{2}{*}{ (b) } & G1 & GAAGTCGTAACAAGG & \multirow{2}{*}{ Jensen et al. [17] } \\
\hline & L1 & CAAGGCATCCACCGT & \\
\hline
\end{tabular}

inner tissues. Serial dilutions and plating were performed as previously described for the analysis of bacteria isolated from the rhizoplane.

Plates were incubated at $28^{\circ} \mathrm{C}$ during $48 \mathrm{~h}$ and after this time total count and count per colony type were performed and expressed as $\mathrm{CFUs} \mathrm{g}^{-1}$ root. All different colony types isolated were purified on NA tubes and incubated at $28^{\circ} \mathrm{C}$ for $48 \mathrm{~h}$.

2.2.2. PCR Amplification of $16 S$ rRNA and 16S-23S rRNA Spacer Sequences. Purified bacterial colonies were placed in Eppendorf tubes with sterile distilled water and $1 \mu \mathrm{l}$ of proteinase $\mathrm{K} 10 \mathrm{mg} \mathrm{ml}^{-1}$. Tubes were incubated $1 \mathrm{~h}$ at $80^{\circ} \mathrm{C}$, $1 \mathrm{~h}$ at $60^{\circ} \mathrm{C}$ and finally, $15 \mathrm{~min}$ at $95^{\circ} \mathrm{C}$. PCR amplications were carried out in $50 \mu \mathrm{L}$ containing $5 \mu \mathrm{l}$ of each cell lysate sample, $5 \mu \mathrm{l}$ of 10x PCR buffer $(500 \mathrm{mM} \mathrm{KCl} ; 100 \mathrm{mM}$ Tris$\mathrm{HCl}, \mathrm{pH} 8.3), 75 \mathrm{mM}$ of $\mathrm{MgCl}_{2}, 10 \mathrm{mM}$ of each dNTP, 15 pmol of each primer, and $1.25 \mathrm{U}$ of Taq polymerase. The primer sets used for the amplification of 16S rRNA and 16S-23S rRNA internal transcribed spacer sequences (ITS) are shown in Table 1 and the position of analyzed sequences within the rRNA operon is presented in Figure 1. Conditions for the amplification of 16S rRNA sequences consisted of an initial denaturation at $94^{\circ} \mathrm{C}(3 \mathrm{~min}), 33$ cycles of amplification at $94^{\circ} \mathrm{C}(45 \mathrm{~s}), 56^{\circ} \mathrm{C}(1 \mathrm{~min})$, and $72^{\circ} \mathrm{C}$ ( $2 \mathrm{~min}$ ), plus one final extension step $5 \mathrm{~min}$ at $72^{\circ} \mathrm{C}$. ITS sequence amplification consisted of an initial denaturation at $94^{\circ} \mathrm{C}(5 \mathrm{~min}), 25$ cycles of amplification at $94^{\circ} \mathrm{C}(1 \mathrm{~min})$, $52^{\circ} \mathrm{C}$ ( $2 \mathrm{~min}$ ), and $72^{\circ} \mathrm{C}(2 \mathrm{~min})$, plus one final extension step $7 \mathrm{~min}$ at $72^{\circ} \mathrm{C}$. Amplifications were performed in a GeneAmp PCR System 9700 (Perkin Elmer).

2.2.3. Electrophoresis and Imaging. Aliquots $(5 \mu \mathrm{l})$ of the different amplified samples were combined with loading buffer (Ficoll 15\%, glycerol 30\%, and 0.25\% xylene cyanol) and the preparations were electrophoresed on $1 \%$ agarose gels in TAE buffer at $100 \mathrm{~V}$ for $1 \mathrm{~h}$. The gels were stained with a solution of ethidium bromide $0.5 \mathrm{mg} \mathrm{L}^{-1}$ [19] and visualized and photographed on a Stratagene Eagle Eye Imaging System. A DNA ladder (0.1-10 Kb; New England BioLabs) was used as the molecular size marker.

2.3. Analysis of Bacterial Diversity by Culture-Independent Methods. The root macerate, obtained after surface-sterilization as previously described, in a sterile chilled mortar was filtered through four sheets of cheesecloth under sterile conditions. A volume of $1 \mathrm{ml}$ of the filtrate was softly poured into chilled centrifuge tubes with Percoll as described for 


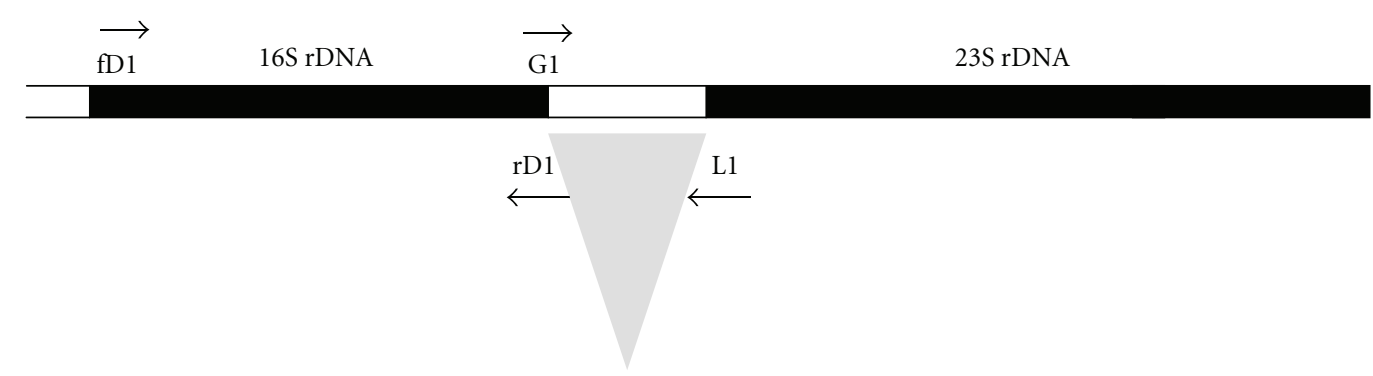

FIGURE 1: Schematic representation of an rRNA operon. Arrows indicate the primer site for PCR amplification of 16S rRNA and 16S-23S rRNA internal transcribed spacer region (ITS).

bacteroid isolation from root nodules by Reibach et al. [20]. The following minor modifications were performed to prepare the gradient: $3.5 \mathrm{ml}$ of $\mathrm{KH}_{2} \mathrm{PO}_{4} 0.5 \mathrm{M}+1.5 \mathrm{M}$ $\mathrm{NaCl}$ were added to chilled centrifuge tubes, followed by $24.5 \mathrm{ml}$ of Percoll and finally $7 \mathrm{ml}$ of sterile distilled water were added to the tubes (final volume: $35 \mathrm{ml}$ ). Tubes were centrifuged at $14000 \mathrm{rpm}$ for $110 \mathrm{~min}$ (Sorvall RC-5B Refrigerated High Speed Centrifuge) and after centrifugation the upper fractions of the gradient were carefully discarded. The bacterial fraction was transferred to clean centrifuge tubes and washed twice with the saline buffer to remove rests of Percoll (4000 rpm for $30 \mathrm{~min}$ ). The bacterial pellet obtained was finally resuspended in the saline buffer, stored at $4^{\circ} \mathrm{C}$ overnight and then used for DNA extraction.

2.4. DNA Extraction and PCR Conditions. Total DNA was extracted directly from the bacterial fraction using GenomicPrep Cells and Tissue DNA Isolation Kit (Amersham), according to the manufacturer's procedure and stored at $-20^{\circ} \mathrm{C}$ until use. PCR amplification of the 16S-23S spacer regions was performed using $\mathrm{G} 1$ and $\mathrm{L} 1$ primers as described for the culture-dependent approach. PCR products were cloned using TOPO-TA Cloning Kit (Invitrogen) and E. coli $\mathrm{DH} 5 \alpha$ chemically competent cells. White colonies were screened for the insertion of ITS sequences using M13 PCR primers. The amplification program consisted of an initial denaturing step at $94^{\circ} \mathrm{C}(3 \mathrm{~min}), 33$ cycles of amplification at $94^{\circ} \mathrm{C}(45 \mathrm{~s}), 48^{\circ} \mathrm{C}(1 \mathrm{~min})$, and $72^{\circ} \mathrm{C}(2 \mathrm{~min})$, plus one final 5 min extension step at $72^{\circ} \mathrm{C}$. All different size bands were sequenced at Macrogen Inc. (Seoul, Korea).

Primers used to amplify 16S rRNA sequences were not used in the culture-independent assay since they presented nonspecific binding to chloroplast sequences. Bacterial identities were confirmed by using both different primer pairs (16S and 16S-23S).

2.5. Analysis of Obtained Sequences. Bacterial taxonomic affiliations were assigned based on the closest match to sequences available at the National Center for Biotechnology Information (NCBI) database (http://www.ncbi.nlm.nih. gov/) using the BLAST algorithm [21]. Sequences of bacteria obtained dependently and independently of culture were deposited in the GenBank nucleotide sequence database (HQ336294-HQ336333).
2.6. Richness and Diversity Indexes. Richness (d) and diversity $(\mathrm{H})$ indexes were calculated based on data corresponding to the total number of bacterial isolates per identified species after sequence analysis. Indexes were also evaluated for bacteria obtained independently of culture by evaluating the total number of clones and the numbers per identified species after sequence analysis, as previously described in Pereira et al. [22], where

$$
\mathrm{d}=\frac{S-1}{\log N}, \quad \mathrm{H}=\frac{C}{N}\left(N \log N-\Sigma n_{i} \log n_{i}\right) .
$$

$S=$ number of different species or groups; $N=$ total number of individuals; $C=2.3 ; n_{i}=$ number of individuals within the $i$ species or group.

\section{Results}

3.1. Total Numbers of Bacterial Isolates. Rhizospheric bacterial populations recovered on NA medium ranged from $9.5 \times$ $10^{6}$ to $3.1 \times 10^{7} \mathrm{CFU} \mathrm{g}^{-1}$ root, while populations recovered from the root inner tissues ranged from $5.0 \times 10^{4}$ to $2.8 \times$ $10^{6} \mathrm{CFU} \mathrm{g}^{-1}$ root (data not shown). Bacterial numbers for each colony type are shown in Table 2.

3.2. Rhizoplane versus Endophytic Bacterial Isolates. According to the culture-dependant approach some bacteria belonging to the Firmicutes, Lysinibacillus and Paenibacillus genera were located exclusively on the rhizoplane of maize seedlings while others such as Achromobacter isolates $(\beta$ Proteobacteria) were recovered from the roots only as endophytes. On the other hand, isolates from Bacillus genus (Firmicutes group) were equally distributed in the external and inner root tissues of maize seedlings. Richness and diversity of culturable bacteria were higher at the rhizoplane $(\mathrm{d}$ index: 6.84, $\mathrm{H}$ index: 2.10 , resp.) than at the root inner tissues (d index: $3.49, \mathrm{H}$ index: 1.48, resp.).

3.3. Culture-Independent versus Culture-Dependent Approach. ITS sequences of representative bacteria isolated from the roots of maize seedlings corresponded to the same taxa as defined by $16 \mathrm{~S}$ rRNA sequences (data not shown), thus validating a comparative analysis. The analysis of ITS sequences obtained by the culture independent approach showed that the largest fraction of the clones of root endophytic bacteria 
TABle 2: Assignment and abundance of 16S rRNA sequences of bacteria isolated (culture-dependent approach) from the rhizoplane and root inner tissues of maize seedlings cv. Monsanto DK684RR2.

\begin{tabular}{|c|c|c|c|c|c|c|c|c|}
\hline Group & Isolate & $\begin{array}{l}\text { Closest NCBI match } \\
\text { Closest type strain }\end{array}$ & $\begin{array}{l}\% \\
\text { Identity }\end{array}$ & $\begin{array}{l}\text { Number of } \\
\text { sequenced } \\
\text { isolates }\end{array}$ & $\begin{array}{l}\text { CFU } g^{-1} \\
\text { fresh root }\end{array}$ & $\begin{array}{l}\% \text { CFU of the } \\
\text { rhizoplane }\end{array}$ & $\begin{array}{l}\% \text { CFU of the } \\
\text { root inner } \\
\text { tissues }\end{array}$ & $\begin{array}{l}\text { Accession } \\
\text { number }\end{array}$ \\
\hline & Bil & $\begin{array}{l}\text { Bacillus sp. GPTSA100-8 } \\
\text { (DQ854983) }\end{array}$ & 98 & 1 & $1.0 \times 10^{5}$ & 0.07 & & HQ336294 \\
\hline & & $\begin{array}{l}\text { Bacillus mycoides strain } \\
\text { ATCC } 6462^{\mathrm{T}}(\mathrm{EF} 210295)\end{array}$ & 97 & & & & & \\
\hline & Bi12 & $\begin{array}{l}\text { Bacillus sp. GPTSA100-8 } \\
\text { (DQ854983) }\end{array}$ & 98 & 1 & $2.0 \times 10^{5}$ & 0.13 & & HQ336295 \\
\hline & & $\begin{array}{l}\text { Bacillus mycoides strain } \\
\text { ATCC } 6462^{\mathrm{T}}(\mathrm{EF} 210295)\end{array}$ & 97 & & & & & \\
\hline & Bi6 & $\begin{array}{l}\text { Bacterium 9-gw 1-9 } \\
\text { (DQ990056) }\end{array}$ & 99 & 1 & $1.0 \times 10^{5}$ & 0.07 & & HQ336296 \\
\hline & & $\begin{array}{l}\text { Bacillus thuringiensis strain } \\
\text { ATCC10792 }(\mathrm{AF} 290545)\end{array}$ & 98 & & & & & \\
\hline & Bi54 & $\begin{array}{l}\text { Bacillus thuringiensis strain } \\
\text { biosZ2 (EU626405) }\end{array}$ & 99 & 1 & $1.6 \times 10^{5}$ & & 2.00 & HQ336297 \\
\hline & & $\begin{array}{l}\text { Bacillus thuringiensis strain } \\
\text { ATCC } 10792^{\mathrm{T}}(\mathrm{AF} 290545)\end{array}$ & 99 & & & & & \\
\hline \multirow[t]{14}{*}{$\begin{array}{l}\text { Firmicutes } \\
(\text { Gram + low } G+C)\end{array}$} & Bi51 & $\begin{array}{l}\text { Bacillus sp. cp-h8 } \\
\text { (EU584532) }\end{array}$ & 100 & 1 & $4.9 \times 10^{6}$ & 2.55 & 11.09 & HQ336298 \\
\hline & & $\begin{array}{l}\text { Bacillus thuringiensis strain } \\
\text { ATCC10792 }(\mathrm{AF} 290545)\end{array}$ & 100 & & & & & \\
\hline & Bi29 & $\begin{array}{l}\text { Bacillus thuringiensis strain } \\
\text { B144 (EU240371) }\end{array}$ & 100 & 7 & $4.8 \times 10^{6}$ & 3.14 & 0.34 & HQ336299 \\
\hline & & $\begin{array}{l}\text { Bacillus thuringiensis strain } \\
\text { ATCC10792 }(\mathrm{AF} 290545)\end{array}$ & 100 & & & & & \\
\hline & Bi23 & $\begin{array}{l}\text { Bacillus cereus strain } \\
\text { IBLO1080 (EU168416) }\end{array}$ & 99 & 1 & $1.0 \times 10^{5}$ & 0.07 & & HQ336300 \\
\hline & & $\begin{array}{l}\text { Bacillus cereus strain ATCC } \\
14579^{\mathrm{T}}\left(\mathrm{NC} \_004722\right)\end{array}$ & 97 & & & & & \\
\hline & Bi59 & $\begin{array}{l}\text { Bacillus sp. B16(A) Ydz-xg } \\
\text { (EU368774) }\end{array}$ & 100 & 10 & $2.2 \times 10^{7}$ & 13.60 & 3.59 & HQ336301 \\
\hline & & $\begin{array}{l}\text { Bacillus megaterium strain } \\
\text { NBRC } 15308^{\mathrm{T}}(\mathrm{AB} 271751)\end{array}$ & 100 & & & & & \\
\hline & Bi19 & $\begin{array}{l}\text { Bacillus simplex strain Q1 } \\
\text { (EU236732) }\end{array}$ & 100 & 6 & $3.1 \times 10^{7}$ & 20.41 & & HQ336302 \\
\hline & & $\begin{array}{l}\text { Bacillus simplex strain LMG } \\
20238^{\mathrm{T}}(\mathrm{AJ} 628748)\end{array}$ & 100 & & & & & \\
\hline & Bi31 & $\begin{array}{l}\text { Uncultured soil bacterium } \\
\text { clone 1296-1 (AF423217) }\end{array}$ & 99 & 1 & $7.0 \times 10^{5}$ & 0.45 & & HQ336303 \\
\hline & & $\begin{array}{l}\text { Bacillus simplex strain LMG } \\
20238^{\mathrm{T}}(\mathrm{AJ} 628748)\end{array}$ & 99 & & & & & \\
\hline & Bi53 & $\begin{array}{l}\text { Bacillus drentensis strain } \\
\text { WN575 (DQ275176) }\end{array}$ & 99 & 1 & $1.0 \times 10^{4}$ & & 0.16 & HQ336304 \\
\hline & & $\begin{array}{l}\text { Bacillus drentensis strain } \\
\text { IDA1967 }{ }^{\mathrm{T}} \text { (NR_029002) }\end{array}$ & 99 & & & & & \\
\hline
\end{tabular}


Table 2: Continued.

\begin{tabular}{|c|c|c|c|c|c|c|c|c|}
\hline Group & Isolate & $\begin{array}{l}\text { Closest NCBI match } \\
\text { Closest type strain }\end{array}$ & $\begin{array}{l}\% \\
\text { Identity }\end{array}$ & $\begin{array}{l}\text { Number of } \\
\text { sequenced } \\
\text { isolates }\end{array}$ & $\begin{array}{l}\text { CFU } g^{-1} \\
\text { fresh root }\end{array}$ & $\begin{array}{l}\% \text { CFU of the } \\
\text { rhizoplane }\end{array}$ & $\begin{array}{l}\% \text { CFU of the } \\
\text { root inner } \\
\text { tissues }\end{array}$ & $\begin{array}{l}\text { Accession } \\
\text { number }\end{array}$ \\
\hline & Bi55 & $\begin{array}{l}\text { Bacillus sp. B222 Ydz-dh } \\
\text { (EU368768) }\end{array}$ & 100 & 2 & $1.2 \times 10^{6}$ & 0.07 & 12.84 & HQ336305 \\
\hline & & $\begin{array}{l}\text { Bacillus pumilus strain } \\
\text { ATCC } 7061^{\mathrm{T}}(\text { AY876289) }\end{array}$ & 100 & & & & & \\
\hline & Bi41 & $\begin{array}{l}\text { Bacillus sp. 3LF21TD } \\
\text { (EU417659) }\end{array}$ & 100 & 1 & $1.0 \times 10^{5}$ & 0.07 & & HQ336306 \\
\hline & & $\begin{array}{l}\text { Bacillus altitudinis strain } \\
41 \mathrm{KF} 2 \mathrm{~b}^{\mathrm{T}} \text { (AJ831842) }\end{array}$ & 100 & & & & & \\
\hline & Bi27 & $\begin{array}{l}\text { Bacillus sp. } 50-3 \\
\text { (EU365432) }\end{array}$ & 100 & 1 & $7.0 \times 10^{6}$ & 4.58 & & HQ336307 \\
\hline & & $\begin{array}{l}\text { Bacillus subtilis subsp } \\
\text { subtilis strain } \\
\text { NBRC } 13719^{\mathrm{T}}(\mathrm{AB} 271744)\end{array}$ & 99 & & & & & \\
\hline & Bi28 & $\begin{array}{l}\text { Bacillus subtilis strain 79-9 } \\
\text { (EU624322) }\end{array}$ & 96 & 1 & $1.0 \times 10^{5}$ & 0.07 & & HQ336308 \\
\hline & & $\begin{array}{l}\text { Bacillus subtilis subsp } \\
\text { subtilis strain } \\
\text { NBRC13719T (AB271744) }\end{array}$ & 96 & & & & & \\
\hline & Bi43 & $\begin{array}{l}\text { Bacillus sp. 12-059 } \\
\text { (EU635727) }\end{array}$ & 100 & 1 & $1.0 \times 10^{5}$ & 0.07 & & HQ336309 \\
\hline & & $\begin{array}{l}\text { Bacillus amyloliquefaciens } \\
\text { strain NBRC } 15535^{\mathrm{T}} \\
(\mathrm{AB} 325583)\end{array}$ & 100 & & & & & \\
\hline & $\mathrm{Bi} 3$ & $\begin{array}{l}\text { Lysinibacillus sphaericus } \\
\text { strain BG-B44 (EU869258) }\end{array}$ & 99 & 2 & $1.3 \times 10^{6}$ & 0.85 & & HQ336310 \\
\hline & & $\begin{array}{l}\text { Lysinibacillus sphaericus } \\
\text { strain NBRC } 15095^{\mathrm{T}} \\
(\mathrm{AB} 271742)\end{array}$ & 98 & & & & & \\
\hline & Bi42 & $\begin{array}{l}\text { Paenibacillus sp. GT08-03 } \\
\text { (AM162320) }\end{array}$ & 99 & 1 & $8.0 \times 10^{5}$ & 0.52 & & HQ336311 \\
\hline & & $\begin{array}{l}\text { Paenibacillus alvei strain } \\
\text { DSM } 29^{\mathrm{T}} \text { (AJ320491) }\end{array}$ & 99 & & & & & \\
\hline \multirow{6}{*}{$\begin{array}{l}\text { Proteobacteria } \beta- \\
\text { Proteobacteria } \\
(\text { Gram }-)\end{array}$} & Bi57 & $\begin{array}{l}\text { Achromobacter xylosoxidans } \\
\text { (DQ414679) }\end{array}$ & 99 & 2 & $5.8 \times 10^{6}$ & & 67.17 & HQ336312 \\
\hline & & $\begin{array}{l}\text { Achromobacter xylosoxidans } \\
\text { strain DSM } 10346^{\mathrm{T}} \\
(\mathrm{Y} 14908)\end{array}$ & 99 & & & & & \\
\hline & \multirow[t]{2}{*}{ Bi65 } & $\begin{array}{l}\text { Achromobacter xylosoxidans } \\
\text { (DQ414679) }\end{array}$ & 100 & 1 & $6.3 \times 10^{4}$ & & 0.61 & HQ336313 \\
\hline & & $\begin{array}{l}\text { Achromobacter xylosoxidans } \\
\text { strain DSM } 10346^{\mathrm{T}} \\
(\mathrm{Y} 14908)\end{array}$ & 99 & & & & & \\
\hline & \multirow[t]{2}{*}{ Bi34 } & $\begin{array}{l}\text { Enterobacter sp. YRL01 } \\
\text { (EU373405) }\end{array}$ & 98 & 2 & $3.3 \times 10^{7}$ & 21.30 & & HQ336314 \\
\hline & & $\begin{array}{l}\text { Enterobacter cloacae subsp. } \\
\text { cloacae strain ATCC } \\
13047^{\mathrm{T}} \text { (NC_014121) }\end{array}$ & 97 & & & & & \\
\hline
\end{tabular}


Table 2: Continued.

\begin{tabular}{|c|c|c|c|c|c|c|c|c|}
\hline Group & Isolate & $\begin{array}{l}\text { Closest NCBI match } \\
\text { Closest type strain }\end{array}$ & $\begin{array}{l}\% \\
\text { Identity }\end{array}$ & $\begin{array}{l}\text { Number of } \\
\text { sequenced } \\
\text { isolates }\end{array}$ & $\begin{array}{l}\text { CFU } g^{-1} \\
\text { fresh root }\end{array}$ & $\begin{array}{l}\% \text { CFU of the } \\
\text { rhizoplane }\end{array}$ & $\begin{array}{l}\% \text { CFU of the } \\
\text { root inner } \\
\text { tissues }\end{array}$ & $\begin{array}{l}\text { Accession } \\
\text { number }\end{array}$ \\
\hline \multirow{14}{*}{$\begin{array}{l}\text { Proteobacteria } \gamma- \\
\text { Proteobacteria } \\
(\text { Gram }-)\end{array}$} & Bi36 & $\begin{array}{l}\text { Enterobacter sp. YRL01 } \\
\text { (EU373405) }\end{array}$ & 97 & 1 & $1.0 \times 10^{5}$ & 0.07 & & HQ336315 \\
\hline & & $\begin{array}{l}\text { Enterobacter cloacae subsp. } \\
\text { cloacae strain ATCC } \\
13047^{\mathrm{T}}(\text { NC_014121) }\end{array}$ & 97 & & & & & \\
\hline & Bi37 & $\begin{array}{l}\text { Enterobacter sp. YRL01 } \\
\text { (EU373405) }\end{array}$ & 99 & 2 & $2.3 \times 10^{7}$ & 14.86 & & HQ336316 \\
\hline & & $\begin{array}{l}\text { Enterobacter cloacae subsp. } \\
\text { cloacae strain ATCC } \\
13047^{\mathrm{T}}(\text { NC_014121) }\end{array}$ & 99 & & & & & \\
\hline & \multirow[t]{2}{*}{ Bi25 } & $\begin{array}{l}\text { Pseudomonas sp. JC1 } \\
\text { (EU704696) }\end{array}$ & 99 & 1 & $4.0 \times 10^{5}$ & 0.26 & & HQ336317 \\
\hline & & $\begin{array}{l}\text { Pseudomonas fluorescens } \\
\text { strain ATCC } \\
13525^{\mathrm{T}}(\mathrm{AJ} 308308)\end{array}$ & 98 & & & & & \\
\hline & \multirow[t]{2}{*}{ Bi60 } & $\begin{array}{l}\text { Pseudomonas fluorescens } \\
\text { strain Mc07 (EF672049) }\end{array}$ & 98 & 2 & $1.8 \times 10^{5}$ & & 2.20 & HQ336318 \\
\hline & & $\begin{array}{l}\text { Pseudomonas fluorescens } \\
\text { strain ATCC } \\
13525^{\mathrm{T}}(\mathrm{AJ} 308308)\end{array}$ & 98 & & & & & \\
\hline & \multirow[t]{2}{*}{ Bi46 } & $\begin{array}{l}\text { Pseudomonas fluorescens } \\
\text { strain Mc07 (EF672049) }\end{array}$ & 96 & 1 & $1.0 \times 10^{5}$ & 0.07 & & HQ336319 \\
\hline & & $\begin{array}{l}\text { Pseudomonas fluorescens } \\
\text { strain ATCC } \\
13525^{\mathrm{T}}(\mathrm{AJ} 308308)\end{array}$ & 95 & & & & & \\
\hline & \multirow[t]{2}{*}{ Bi44 } & $\begin{array}{l}\text { Pseudomonas putida strain } \\
\text { PC16 (AY918067) }\end{array}$ & 99 & 2 & $1.1 \times 10^{7}$ & 7.33 & & HQ336320 \\
\hline & & $\begin{array}{l}\text { Pseudomonas putida strain } \\
\text { ATCC } 12633^{\mathrm{T}} \text { (AF094736) }\end{array}$ & 96 & & & & & \\
\hline & \multirow[t]{2}{*}{ Bi49 } & $\begin{array}{l}\text { Pseudomonas putida strain } \\
\text { PC16 (AY918067) }\end{array}$ & 98 & 2 & $1.4 \times 10^{7}$ & 9.39 & & HQ336321 \\
\hline & & $\begin{array}{l}\text { Pseudomonas putida strain } \\
\text { ATCC } 12633^{\mathrm{T}} \text { (AF094736) }\end{array}$ & 96 & & & & & \\
\hline
\end{tabular}

${ }^{\mathrm{T},}$ Type strain.

belonged to $\gamma$-Proteobacteria (79.5\%), with Enterobacter, Klebsiella, Erwinia and Pseudomonas as prevalent bacterial genera identified. The remaining 20.5 percent of the clones belonged to Bacillus (Table 3 ). Richness index (d) calculated for the clones was 3.56 while diversity index $(\mathrm{H})$ was 1.81 .

According to the bacterial community composition associated with the roots of maize seedlings assessed by culture, $68 \%$ of total CFUs belonged to $\beta$-Proteobacteria (Achromobacter), 30\% to Firmicutes (Bacillus), and only 2\% were identified as $\gamma$-Proteobacteria (Pseudomonas) (Table 2). Within the group of endophytic bacteria $81.5 \%$ of the isolates belonged to Bacillus genus, $11.1 \%$ to Achromobacter and the remaining $7.4 \%$ was identified as Pseudomonas.
Bacterial richness $(\mathrm{d})$ and diversity $(\mathrm{H})$ indexes calculated for endophytic culturable bacteria were slightly lower than the ones obtained in the culture-independent approach (3.49 and 1.48 , resp. versus 3.56 and 1.81).

\section{Discussion}

In the present work culture dependent and culture independent methods were applied to analyze bacterial diversi-ty associated with the roots of maize seedlings. Most frequently reported bacteria linked to the maize crop (Zea mays L.) are Firmicutes (Bacillus), $\gamma$-Proteobacteria (Pseudomonas, 
TABLE 3: Assignment and abundance of ITS clones (culture-independent approach) of bacterial endophytes of maize seedlings cv. Monsanto DK684RR2.

\begin{tabular}{|c|c|c|c|c|c|c|c|}
\hline Group & Clone & Closest NCBI match & $\begin{array}{l}\% \\
\text { Identity }\end{array}$ & $\begin{array}{l}\% \text { sequence } \\
\text { coverage }\end{array}$ & $\begin{array}{l}\mathrm{N}^{\circ} \\
\text { clones }\end{array}$ & $\begin{array}{l}\% \\
\text { clones }\end{array}$ & $\begin{array}{l}\text { Accession } \\
\text { number }\end{array}$ \\
\hline \multirow{4}{*}{$\begin{array}{l}\text { Firmicutes } \\
(\text { Gram + low G + C) }\end{array}$} & Bs6 & $\begin{array}{l}\text { Bacillus thuringiensis serovar konkukian } \\
\text { strain 97-27 (AE017355) }\end{array}$ & 98 & 100 & 3 & 3.24 & HQ336322 \\
\hline & \multirow{2}{*}{ Bs27 } & Bacillus sp. CP8 (GU905014) & 99 & 100 & \multirow{2}{*}{11} & \multirow{2}{*}{11.87} & \multirow{2}{*}{ HQ336323 } \\
\hline & & Bacillus mycoides strain 4749 (GQ255890) & 98 & 100 & & & \\
\hline & Bs28 & $\begin{array}{l}\text { Bacillus amyloliquefaciens FZB42 } \\
\text { (CP000560) }\end{array}$ & 100 & 100 & 5 & 5.39 & HQ336324 \\
\hline \multirow{12}{*}{$\begin{array}{l}\gamma \text {-Proteobacteria } \\
(\text { Gram }-)\end{array}$} & \multirow{2}{*}{ Bs2 } & Uncultured bacterium clone 6 (DQ011253) & 92 & 100 & \multirow{2}{*}{6} & \multirow{2}{*}{6.45} & \multirow{2}{*}{ HQ336325 } \\
\hline & & Klebsiella oxytoca strain g1755 (EU623326) & 92 & 100 & & & \\
\hline & Bs17 & Klebsiella variicola At-22 (СР001891) & 97 & 100 & 8 & 8.60 & HQ336326 \\
\hline & Bs85 & $\begin{array}{l}\text { Klebsiella pneumoniae subsp. pneumoniae } \\
\text { MGH78578 (CP000647) }\end{array}$ & 95 & 100 & 2 & 2.16 & HQ336327 \\
\hline & Bs89 & Klebsiella oxytoca strain g1755 (EU623329) & 92 & 100 & 1 & 1.08 & HQ336328 \\
\hline & Bs43 & $\begin{array}{l}\text { Erwinia rhapontici strain ATCC } 29283 \\
\text { (AF232678) }\end{array}$ & 92 & 100 & 7 & 7.54 & HQ336329 \\
\hline & Bs44 & Erwinia pyrifoliae strain WT10 (EF422400) & 90 & 100 & 5 & 5.38 & HQ336330 \\
\hline & \multirow[t]{2}{*}{ Bs83 } & Uncultured bacterium clone 7 (DQ011254) & 96 & 100 & \multirow[t]{2}{*}{27} & \multirow{2}{*}{28.94} & \multirow{2}{*}{ HQ336331 } \\
\hline & & $\begin{array}{l}\text { Enterobacter cloacae subsp. cloacae ATCC } \\
13047 \text { (CP001918) }\end{array}$ & 94 & 100 & & & \\
\hline & \multirow[t]{2}{*}{ Bs54 } & $\begin{array}{l}\text { Uncultured Stenotrophomonas sp. clone } \\
\text { 5TC3 (GQ228658) }\end{array}$ & 98 & 100 & \multirow[t]{2}{*}{17} & \multirow[t]{2}{*}{18.27} & \multirow[t]{2}{*}{ HQ336332 } \\
\hline & & $\begin{array}{l}\text { Stenotrophomonas maltophilla K279a } \\
\text { (AM743169) }\end{array}$ & 97 & 100 & & & \\
\hline & Bs68 & Pseudomonas tolaasii LMG 2342 (AF364307) & 96 & 100 & 1 & 1.08 & HQ336333 \\
\hline
\end{tabular}

Enterobacter, Pantoea, and Klebsiella), $\beta$-Proteobacteria (Burkholderia), $\alpha$-Proteobacteria (Rhizobium), and Actinobacteria (Arthrobacter). Less frequent are Paenibacillus (Firmi-cutes), Achromobacter, Herbaspirillum ( $\beta$-Proteobacteria), Erwinia ( $\gamma$-Proteobacteria), Sphingomonas ( $\alpha$-Proteobacteria), other Actinobacteria, and Bacteroidetes [18, 2325]. In agreement with these previous surveys, within the Firmicutes group we identified Bacillus species from both the rhizoplane and root inner tissues of maize seedlings and Enterobacter and Pseudomonas were found as main genera within the $\gamma$-Proteobacteria. Bacteria from Bacillus genus have been reported as maize kernel endophytes [10] and have been isolated not only from maize $[10,18,26]$ but also from many different plants such as peas, potatoes, conifers, bananas, and bean [27-31]. Their activities as biocontrol agents of various fungi $[32,33]$, as phosphorous solubilizers [34] and as auxin producers [35] have also been reported.

Bacillus and Pseudomonas were previously identified as major genera isolated from 20-day-old maize seedlings using the same cultivar (DK864RR2) and identified based on morphophysiological analysis [8], thus indicating correspondence between morphophysiological and genetic approaches (Table 4).

Achromobacter xylosoxidans, the most abundant species from the root inner tissues of DK684RR2 maize seedlings in the culture dependent approach, has been found previously associated with maize, promoting mycotoxin synthesis inhibition [36] and has also been reported as a growth promoting bacteria of Brassica juncea [37]. The fact that A. xylosoxidans was isolated from the inner tissues of maize seedlings suggest that this bacterium is kernel borne.

Paenibacillus was a minor isolate in our work. It has been previously isolated from the rhizosphere of maize [38] and wheat [39] and has been also found as an endophyte of Eucalyptus, soybean, and Phaseolus vulgaris beans [31, 33, 40].

Estimated bacterial numbers here are within the ranges with the ones reported for maize endophytes in previous surveys $[23,24,26]$.

Not all bacterial genera identified by the culture dependent approach were recovered by the culture independent method. Culture-independent methods may be affected by the heterogeneous lysis of different bacterial species and may suffer from primer bias however they supposedly provide more complete data of the bacterial community including viable but not culturable bacteria (VNC) and also nonviable bacteria (NV). Underrepresentation of VNC and NV in cultures is expected and could explain the differences observed with both approaches. Maybe many $\gamma$-Proteobacteria remain non culturable. It has been previously reported that some endophytic bacteria can attain a VNC state [41]. On the other hand, it is known that some Bacillus isolates produce a wide spectrum of antibiotics such as bacillomicin, megacine, fengicine, iturin, mycosubtilin and zwittermicin 
TABLE 4: Frequency (\% of CFU or clones) of different bacterial genera associated with the rhizoplane and root inner tissues of maize seedlings cv. Monsanto DK684RR2.

\begin{tabular}{|c|c|c|c|c|}
\hline \multirow[b]{2}{*}{ Group } & \multirow[b]{2}{*}{ Genera } & \multicolumn{2}{|c|}{ Culture-dependent } & \multirow{2}{*}{$\begin{array}{c}\text { Culture-independent } \\
\text { Endophytes }\end{array}$} \\
\hline & & Rhizoplane & Endophytes & \\
\hline \multirow{3}{*}{ Firmicutes } & Bacillus & 45.35 & 30.02 & 20.43 \\
\hline & Lysinibacillus & 0.85 & & \\
\hline & Paenibacillus & 0.52 & & \\
\hline$\beta$-Proteobacteria & Achromobacter & & 67.78 & \\
\hline \multirow{5}{*}{$\gamma$-Proteobacteria } & Pseudomonas & 17.05 & 2.2 & 1.08 \\
\hline & Enterobacter & 36.23 & & 29.03 \\
\hline & Klebsiella & & & 18.28 \\
\hline & Erwinia & & & 12.90 \\
\hline & Stenotrophomonas & & & 18.28 \\
\hline
\end{tabular}

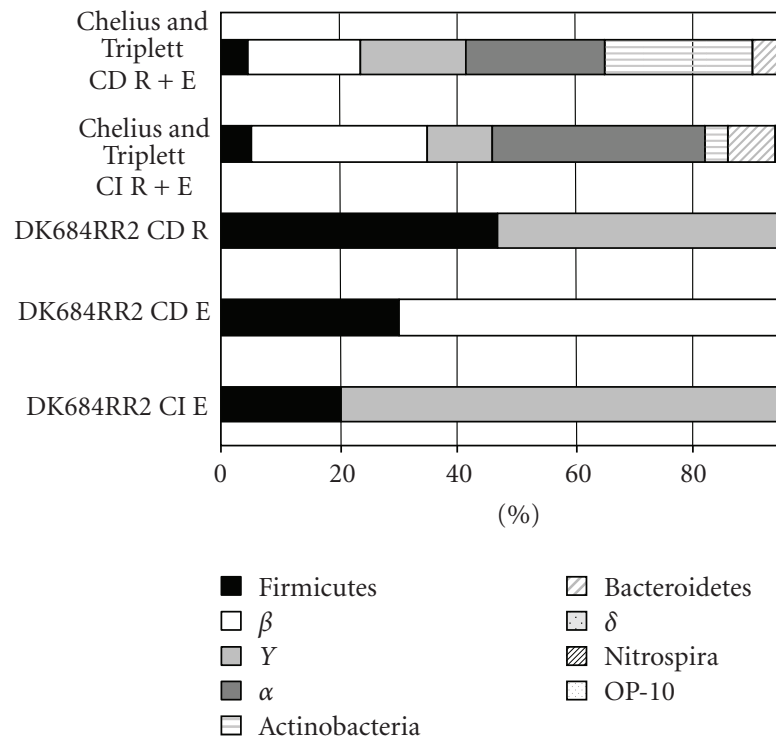

FIGURE 2: Community composition of bacteria based on culture dependent (CD) and culture-independent methods (CI) from maize cv. Monsanto DK684RR2. Chelius and Triplett [18] results were added for comparison. The Greek letters correspond to Proteobacteria subdivisions. R: rhizoplane; E: endophytes; R + E: rhizoplane plus endophytes.

that may possibly inhibit growth of other bacteria in plates. In addition, some Pseudomonas isolates may produce 2,4diacetilfluoroglucinol, fenacine and pirrolnitrine that may restrict even more the spectrum of microorganisms able to grow in culture.

Gomes et al. [42] also found Proteobacteria as the major group when studying maize rhizospheric bacteria independently of culture. Schmalenberger and Tebbe [14] have also stated that most bacterial sequences obtained from the maize rhizoplane by direct DNA extraction from roots belonged to Proteobacteria. Sun et al. [43] found $\beta$-Proteobacteria as the dominant group in root inner tissues when studying rice-associated bacteria, as we did by the culture dependent approach.
Enterobacter has been found previously as an endophyte of maize $[10,26]$ and other plants such as rice, cotton, papaya and poplar [44-47] and its ability to antagonize maize fungal pathogens within Fusarium genus has also been described [48]. Stenotrophomonas has not been reported before in association with maize, but it was found as the most abundant genus present in rice [43]. Endophytic Stenotrophomonas were also obtained from potato, coffee and poplar $[28,46,49]$.

We detected here a different bacterial community profile than the one reported by Chelius and Triplett [18] when studying culturable and not culturable bacteria associated with the roots of physiologically mature maize plants (Figure 2). Differences may be explained by variables such as the plant age (102 instead of 20-day-old plants in this study), other conditions related to the growth of plants such as fertilization used in comparison to no fertilization in this work, and the plant material used (cv. Pioneer 3751 instead of cv. Monsanto DK684RR2). It has been reported that the plant genotype and age markedly influence the profile of associated bacteria $[42,50-54]$.

In a recent review on endophytes, it is considered that culture dependent and independent approaches are complementary [55]. Current methods available for the analysis of the effects of different microorganisms on a particular crop rely on isolation and consequently on culture. The knowledge of the wide spectrum of maize associated bacteria will facilitate the search of bacteria capable of exerting antagonism to pathogenic infections, or the detection of biological plant growth enhancers. A new view of maize associated bacteria could be obtained in the future by metagenomic and functional metagenomic analyses and results derived from culture independent approaches like the one performed in the present work could be a basis for such studies.

\section{Acknowledgment}

The authors thank J. Quiroz-López for technical help, DGAPA for PAPIIT Grant IN200709-3, UNAM, México, and CONICET PIP 5822/05 from Argentina. 


\section{References}

[1] J. von Braun, "The world food situation. New driving forces and required actions," 2010, http://assets.mediaglobal.org/ documents/World_Food_Situation_New_Driving_Forces_and_ Required_Action_Released_by_the_International_Food_Policy_ Institute.pdf.

[2] J. Zhou, B. Xia, H. Huang et al., "Bacterial phylogenetic diversity and a novel candidate division of two humid region, sandy surface soils," Soil Biology and Biochemistry, vol. 35, no. 7, pp. 915-924, 2003.

[3] G. V. Bloemberg and B. J. J. Lugtenberg, "Molecular basis of plant growth promotion and biocontrol by rhizobacteria," Current Opinion in Plant Biology, vol. 4, no. 4, pp. 343-350, 2001.

[4] E. A. B. Emmert and JO. Handelsman, "Biocontrol of plant disease: a $\left(\mathrm{Gram}^{+}\right)$positive perspective," FEMS Microbiology Letters, vol. 171, no. 1, pp. 1-9, 1999.

[5] J. D. Oliver, "Recent findings on the viable but nonculturable state in pathogenic bacteria," FEMS Microbiology Reviews, vol. 34 , no. 4, pp. 415-425, 2010.

[6] V. Torsvik, L. Øvreås, and T. F. Thingstad, "Prokaryotic diversity-magnitude, dynamics, and controlling factors," Science, vol. 296, no. 5570, pp. 1064-1066, 2002.

[7] A. Sessitsch, B. Reiter, and G. Berg, "Endophytic bacterial communities of field-grown potato plants and their plantgrowth-promoting and antagonistic abilities," Canadian Journal of Microbiology, vol. 50, no. 4, pp. 239-249, 2004.

[8] P. Pereira, A. Nesci, and M. Etcheverry, "Impact of two bacterial biocontrol agents on bacterial and fungal culturable groups associated with the roots of field-grown maize," Letters in Applied Microbiology, vol. 48, no. 4, pp. 493-499, 2009.

[9] R. Rai, P. K. Dash, B. M. Prasanna, and A. Singh, "Endophytic bacterial flora in the stem tissue of a tropical maize (Zea mays L.) genotype: isolation, identification and enumeration," World Journal of Microbiology and Biotechnology, vol. 23, no. 6, pp. 853-858, 2007.

[10] T. Rijavec, A. Lapanje, M. Dermastia, and M. Rupnik, "Isolation of bacterial endophytes from germinated maize kernels," Canadian Journal of Microbiology, vol. 53, no. 6, pp. 802-808, 2007.

[11] Y. Herschkovitz, A. Lerner, Y. Davidov et al., "Inoculation with the plant-growth-promoting rhizobacterium Azospirillum brasilense causes little disturbance in the rhizosphere and rhizoplane of maize (Zea mays)," Microbial Ecology, vol. 50, no. 2, pp. 277-288, 2005.

[12] H. Sanguin, A. Herrera, C. Oger-Desfeux et al., "Development and validation of a prototype $16 \mathrm{~S}$ rRNA-based taxonomic microarray for Alphaproteobacteria," Environmental Microbiology, vol. 8, no. 2, pp. 289-307, 2006.

[13] H. Sanguin, B. Remenant, A. Dechesne et al., "Potential of a $16 S$ rRNA-based taxonomic microarray for analyzing the rhizosphere effects of maize on Agrobacterium spp. and bacterial communities," Applied and Environmental Microbiology, vol. 72, no. 6, pp. 4302-4312, 2006.

[14] A. Schmalenberger and C. C. Tebbe, "Bacterial diversity in maize rhizospheres: conclusions on the use of genetic profiles based on PCR-amplified partial small subunit rRNA genes in ecological studies," Molecular Ecology, vol. 12, no. 1, pp. 251262,2003

[15] L. Miché and J. Balandreau, "Effects of rice seed surface sterilization with hypochlorite on inoculated Burkholderia vietnamiensis," Applied and Environmental Microbiology, vol. 67, no. 7, pp. 3046-3052, 2001.
[16] W. G. Weisburg, S. M. Barns, D. A. Pelletier, and D. J. Lane, "16S ribosomal DNA amplification for phylogenetic study," Journal of Bacteriology, vol. 173, no. 2, pp. 697-703, 1991.

[17] M. A. Jensen, J. A. Webster, and N. Straus, "Rapid identification of bacteria on the basis of polymerase chain reactionamplified ribosomal DNA spacer polymorphisms," Applied and Environmental Microbiology, vol. 59, no. 4, pp. 945-952, 1993.

[18] M. K. Chelius and E. W. Triplett, "The diversity of archaea and bacteria in association with the roots of Zea mays L," Microbial Ecology, vol. 41, no. 3, pp. 252-263, 2001.

[19] J. Sambrook, E. F. Fritsch, and T. Maniatis, Molecular Cloning: A Laboratory Manual, Cold Spring Harbor Laboratory, Cold Spring Harbor Laboratory Press, New York, NY, USA, 2nd edition, 1989.

[20] P. H. Reibach, P. L. Mask, and J. G. Streeter, "A rapid onestep method for the isolation of bacteroids from root nodules of soybean plants, utilizing self-generating Percoll gradients," Canadian Journal of Microbiology, vol. 27, no. 5, pp. 491-495, 1981.

[21] S. F. Altschul, T. L. Madden, A. A. Schäffer et al., "Gapped BLAST and PSI-BLAST: a new generation of protein database search programs," Nucleic Acids Research, vol. 25, no. 17, pp. 3389-3402, 1997.

[22] P. Pereira, A. Nesci, and M. G. Etcheverry, "Efficacy of bacterial seed treatments for the control of Fusarium verticillioides in maize," BioControl, vol. 54, no. 1, pp. 103-111, 2009.

[23] L. Brusetti, P. Francia, C. Bertolini et al., "Bacterial communities associated with the rhizosphere of transgenic $\mathrm{Bt} 176$ maize (Zea mays) and its non transgenic counterpart," Plant and Soil, vol. 266, no. 1-2, pp. 11-21, 2005.

[24] P. J. Fisher, O. Petrini, and H. M. L. Scott, "The distribution of some fungal and bacterial endophytes in maize (Zea mays L.)," New Phytologist, vol. 122, no. 2, pp. 299-305, 1992.

[25] L. F. W. Roesch, L. M. P. Passaglia, F. M. Bento, E. W. Triplett, and F. A. O. Camargo, "Diversity of diazotrophic endophytic bacteria associated with maize plants," Revista Brasileira de Ciencia do Solo, vol. 31, no. 6, pp. 1367-1380, 2007.

[26] J. A. McInroy and J. W. Kloepper, "Survey of indigenous bacterial endophytes from cotton and sweet corn," Plant and Soil, vol. 173, no. 2, pp. 337-342, 1995.

[27] M. Elvira-Recuenco and J. W. L. van Vuurde, "Natural incidence of endophytic bacteria in pea cultivars under field conditions," Canadian Journal of Microbiology, vol. 46, no. 11, pp. 1036-1041, 2000.

[28] P. Garbeva, L. S. van Overbeek, J. W. L. van Vuurde, and J. D. van Elsas, "Analysis of endophytic bacterial communities of potato by plating and denaturing gradient gel electrophoresis (DGGE) of $16 \mathrm{~S}$ rDNA based PCR fragments," Microbial Ecology, vol. 41, no. 4, pp. 369-383, 2001.

[29] H. Izumi, I. C. Anderson, K. Killham, and E. R. B. Moore, "Diversity of predominant endophytic bacteria in European deciduous and coniferous trees," Canadian Journal of Microbiology, vol. 54, no. 3, pp. 173-179, 2008.

[30] J. Lian, Z. Wang, and S. Zhou, "Response of endophytic bacterial communities in banana tissue culture plantlets to Fusarium wilt pathogen infection," Journal of General and Applied Microbiology, vol. 54, no. 2, pp. 83-92, 2008.

[31] A. López-López, M. A. Rogel, E. Ormeño-Orrillo, J. MartínezRomero, and E. Martínez-Romero, "Phaseolus vulgaris seedborne endophytic community with novel bacterial species such as Rhizobium endophyticum sp. nov.," Systematic and Applied Microbiology, vol. 33, pp. 322-327, 2010. 
[32] R. Aravind, A. Kumar, S. J. Eapen, and K. V. Ramana, "Endophytic bacterial flora in root and stem tissues of black pepper (Piper nigrum L.) genotype: isolation, identification and evaluation against Phytophthora capsici," Letters in Applied Microbiology, vol. 48, no. 1, pp. 58-64, 2009.

[33] M. Senthilkumar, K. Swarnalakshmi, V. Govindasamy, Y. K. Lee, and K. Annapurna, "Biocontrol potential of soybean bacterial endophytes against charcoal rot fungus, Rhizoctonia bataticola," Current Microbiology, vol. 58, no. 4, pp. 288-293, 2009.

[34] R. C. Boro, C. Goswami, D. Thakuria, M. K. Modi, and N. C. Talukdar, "Molecular and functional characteristics, growth promoting effect and persistence of selected parent isolates and streptomycin resistant derivatives of rice rhizobacteria," Indian Journal of Experimental Biology, vol. 42, no. 12, pp. 1186-1194, 2004.

[35] M. Park, C. Kim, J. Yang et al., "Isolation and characterization of diazotrophic growth promoting bacteria from rhizosphere of agricultural crops of Korea," Microbiological Research, vol. 160, no. 2, pp. 127-133, 2005.

[36] P. S. Yan, Y. Song, E. Sakuno, H. Nakajima, H. Nakagawa, and K. Yabe, "Cyclo(L-leucyl-L-prolyl) produced by Achromobacter xylosoxidans inhibits aflatoxin production by Aspergillus parasiticus," Applied and Environmental Microbiology, vol. 70, no. 12, pp. 7466-7473, 2004.

[37] Y. Ma, M. Rajkumar, and H. Freitas, "Inoculation of plant growth promoting bacterium Achromobacter xylosoxidans strain Ax10 for the improvement of copper phytoextraction by Brassica juncea," Journal of Environmental Management, vol. 90, no. 2, pp. 831-837, 2009.

[38] I. Von Der Weid, E. Paiva, A. Nóbrega, J. Dirk Van Elsas, and L. Seldin, "Diversity of Paenibacillus polymyxa strains isolated from the rhizosphere of maize planted in Cerrado soil," Research in Microbiology, vol. 151, no. 5, pp. 369-381, 2000.

[39] A. Beneduzi, D. Peres, P. B. da Costa, M. H. Bodanese Zanettini, and L. M. P. Passaglia, "Genetic and phenotypic diversity of plant-growth-promoting bacilli isolated from wheat fields in southern Brazil," Research in Microbiology, vol. 159, no. 4, pp. 244-250, 2008.

[40] A. Ferreira, M. C. Quecine, P. T. Lacava, S. Oda, J. L. Azevedo, and W. L. Araújo, "Diversity of endophytic bacteria from Eucalyptus species seeds and colonization of seedlings by Pantoea agglomerans," FEMS Microbiology Letters, vol. 287, no. 1, pp. 8-14, 2008.

[41] T. Hurek, L. L. Handley, B. Reinhold-Hurek, and Y. Piché, "Azoarcus grass endophytes contribute fixed nitrogen to the plant in an unculturable state," Molecular Plant-Microbe Interactions, vol. 15, no. 3, pp. 233-242, 2002.

[42] N. C. M. Gomes, H. Heuer, J. Schönfeld, R. Costa, L. Mendonça-Hagler, and K. Smalla, "Bacterial diversity of the rhizosphere of maize (Zea mays) grown in tropical soil studied by temperature gradient gel electrophoresis," Plant and Soil, vol. 232, no. 1-2, pp. 167-180, 2001.

[43] L. Sun, F. Qiu, X. Zhang, X. Dai, X. Dong, and W. Song, "Endophytic bacterial diversity in rice (Oryza sativa L.) roots estimated by $16 \mathrm{~S}$ rDNA sequence analysis," Microbial Ecology, vol. 55, no. 3, pp. 415-424, 2008.

[44] A. Elbeltagy, K. Nishioka, T. Sato et al., "Endophytic colonization and in planta nitrogen fixation by a Herbaspirillum sp. Isolated from wild rice species," Applied and Environmental Microbiology, vol. 67, no. 3-12, pp. 5285-5293, 2001.
[45] A. Quadt-Hallmann and J. W. Kloepper, "Immunological detection and localization of the cotton endophyte Enterobacter asburiae JM22 in different plant species," Canadian Journal of Microbiology, vol. 42, no. 11, pp. 1144-1154, 1996.

[46] S. Taghavi, C. Garafola, S. Monchy et al., "Genome survey and characterization of endophytic bacteria exhibiting a beneficial effect on growth and development of poplar," Applied and Environmental Microbiology, vol. 75, no. 3, pp. 748-757, 2009.

[47] P. Thomas, S. Kumari, G. K. Swarna, and T. K. S. Gowda, "Papaya shoot tip associated endophytic bacteria isolated from in vitro cultures and host-endophyte interaction in vitro and in vivo," Canadian Journal of Microbiology, vol. 53, no. 3, pp. 380-390, 2007.

[48] D. M. Hinton and C. W. Bacon, "Enterobacter cloacae is an endophytic symbiont of corn," Mycopathologia, vol. 129, no. 2, pp. 117-125, 1995.

[49] F. E. Vega, M. Pava-Ripoll, F. Posada, and J. S. Buyer, "Endophytic bacteria in Coffea arabica L," Journal of Basic Microbiology, vol. 45, no. 5, pp. 371-380, 2005.

[50] E. Baudoin, E. Benizri, and A. Guckert, "Impact of growth stage on the bacterial community structure along maize roots, as determined by metabolic and genetic fingerprinting," Applied Soil Ecology, vol. 19, no. 2, pp. 135-145, 2002.

[51] G. Granér, P. Persson, J. Meijer, and S. Alström, "A study on microbial diversity in different cultivars of Brassica napus in relation to its wilt pathogen, Verticillium longisporum," FEMS Microbiology Letters, vol. 224, no. 2, pp. 269-276, 2003.

[52] J. Hallman and G. Berg, "Spectrum and population dynamics of bacterial root endophytes," in Microbial Root Endophytes, B. J. E. Schulz, C. J. C. Boyle, and T. N. Sieber, Eds., pp. 15-31, Springer, New York, NY, USA, 2006.

[53] M. Rosenblueth and E. Martínez-Romero, "Rhizobium etli maize populations and their competitivenes for roof colonization," Archives of Microbiology, vol. 181, no. 5, pp. 337-344, 2004.

[54] A. V. Sturz, B. R. Christie, B. G. Matheson, W. J. Arsenault, and N. A. Buchanan, "Endophytic bacterial communities in the periderm of potato tubers and their potential to improve resistance to soil-borne plant pathogens," Plant Pathology, vol. 48, no. 3, pp. 360-369, 1999.

[55] P. R. Hardoim, L. S. van Overbeek, and J. D. V. van Elsas, "Properties of bacterial endophytes and their proposed role in plant growth," Trends in Microbiology, vol. 16, no. 10, pp. 463-471, 2008. 

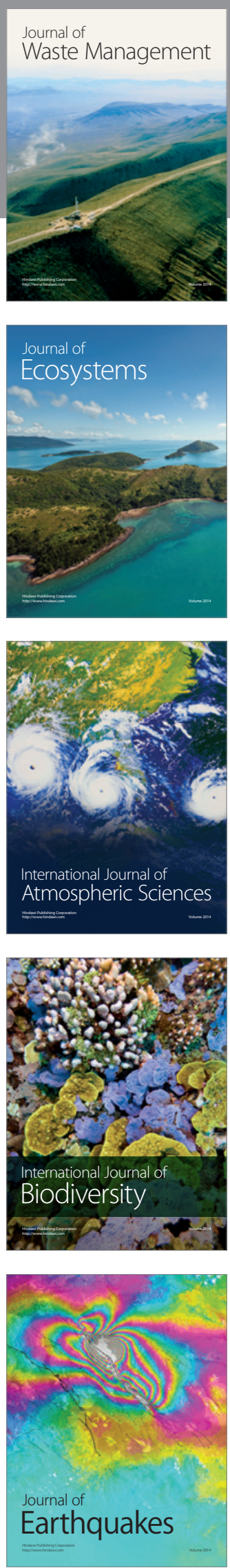
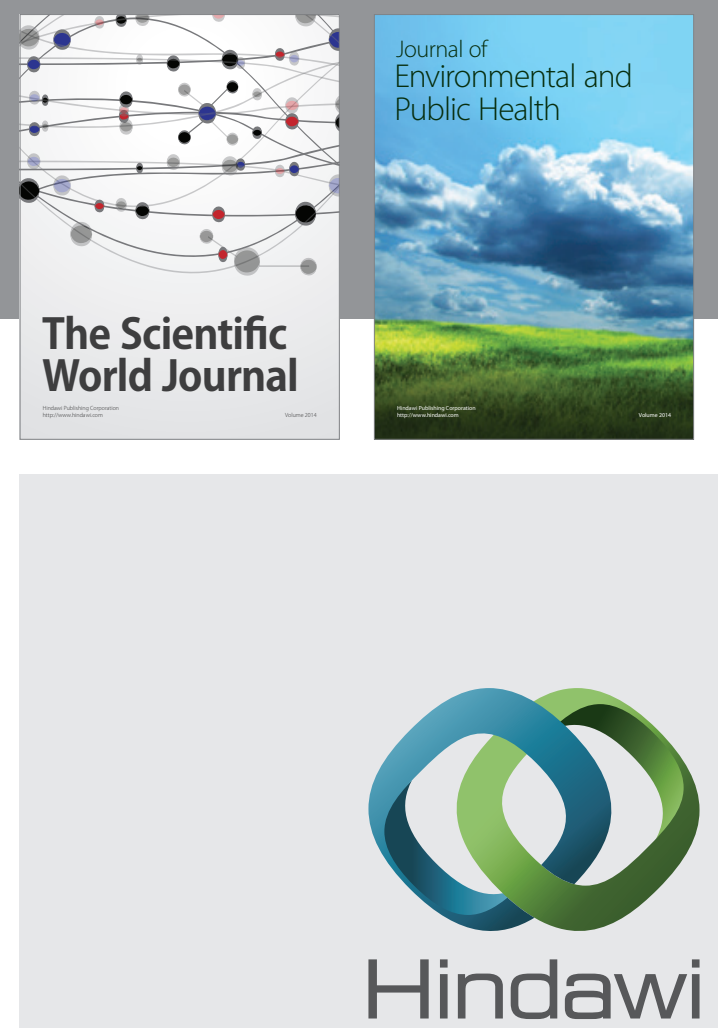

Submit your manuscripts at

http://www.hindawi.com
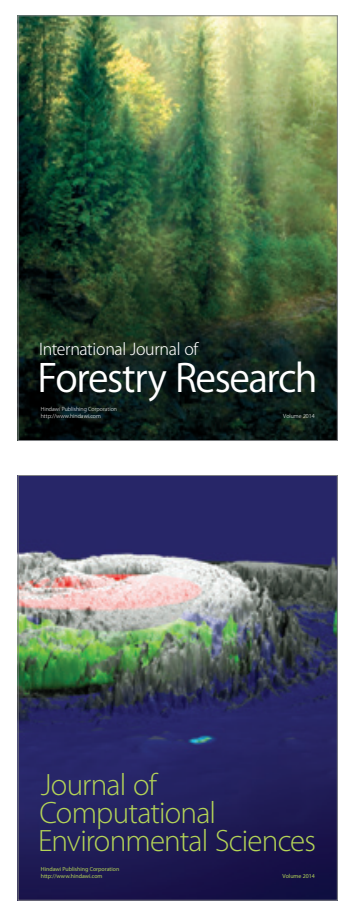
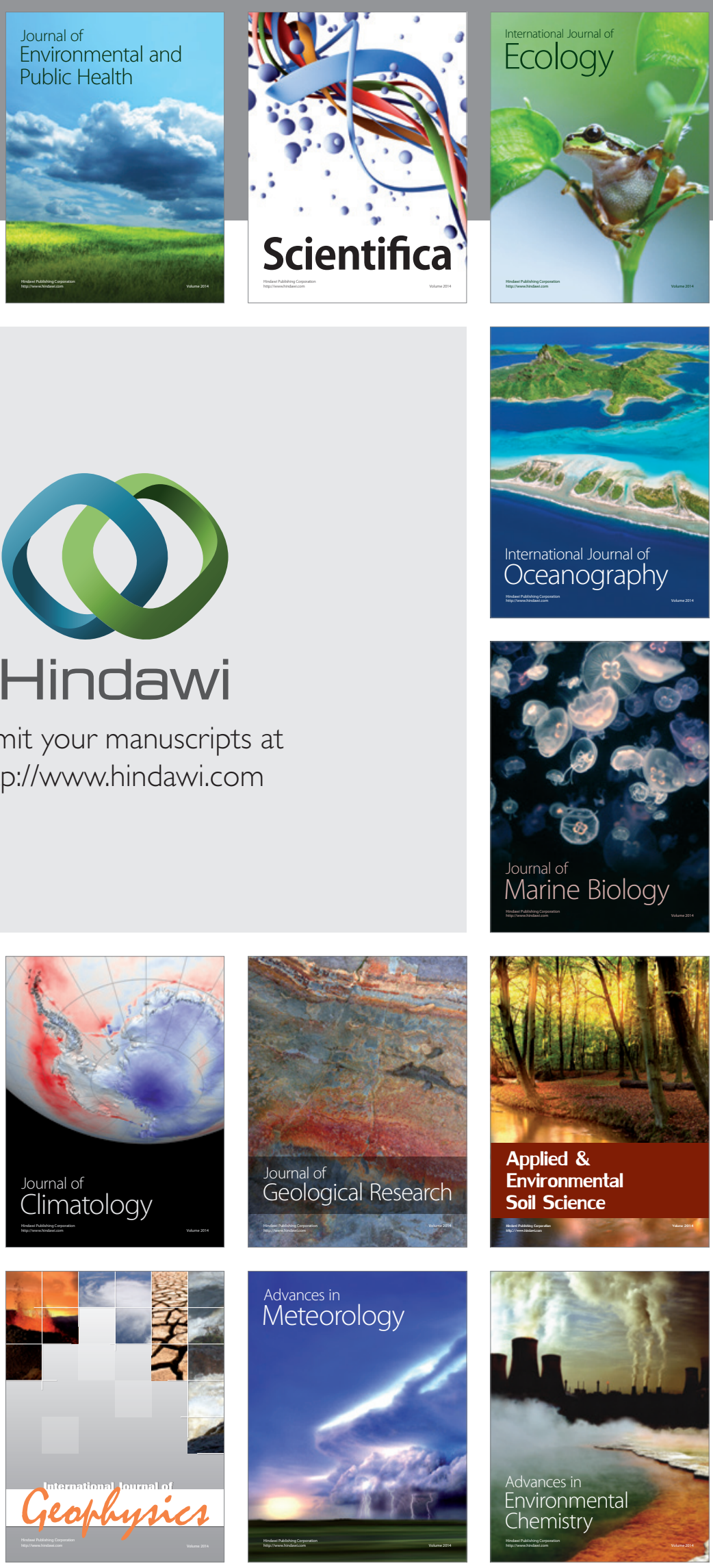